\title{
Analyse De L'état De La Diversité Floristique Des Plateaux Suivant Un Gradient D'aridité Nord- Sud Dans La Réserve De Biosphère Du W Du Niger
}

\author{
Mamadou Ä̈ssa Jazy \\ Laboratoire de Botanique- Pharmacognosie, Faculté des Sciences de la Santé, \\ Université Abdou Moumouni, Niamey-Niger
}

Soumana Douma

Laboratoire de Biologie Garba Mounkaila, Département de Biologie, Faculté des Sciences et Techniques, Université Abdou Moumouni,

Niamey, Niamey-Niger.

\section{Ali Mahamane}

Université de Diffa, Faculté des Sciences Agronomiques, Diffa-Niger

\section{Saadou Mahamane}

Laboratoire de Biologie, Faculté des Sciences et Techniques, Université Dan Dicko Dankoulodo, Maradi-Niger.

\section{Doi: 10.19044/esj.2018.v14n3p156 URL:http://dx.doi.org/10.19044/esj.2018.v14n3p156}

\begin{abstract}
Introduction: In Niger, the degradation of ecosystems leads to a loss of biodiversity and an increase in the adverse effects of climate change, especially in the ecosystems contracted from the plateaux of the Niger W biosphere reserve. Objective: The objective of this study is to characterize the spatial variability of the flora according to the north-south rainfall gradient of the reserve. Methodology: Phytosociological surveys, an ascending hierarchical classification and a canonical analysis of correspondences between plant groups and environmental factors. Results-Discussion: The 126 inventoried species have 88 dicotyledons, 28 woody species, and 98 herbaceous species. The most common families are: Poaceae $(21.42 \%)$, Fabaceae (12.69\%), Rubiaceae (7.14\%) and Convolvulaceae $(6.34 \%)$. The biological spectrum is dominated by therophytes then microphanerophytes. The world chorology is marked by African species followed by palaeotropics or pantropicales, and at the African level, Sudano-Zambezian species are the most frequent. The presence of intermediate species (GC-SZ and GC-SZ-Sah$S$ ) is noted. From north to south, 3 groups of plants were highlighted: the group with Sida cordifolia and Guiera senegalensis whose phytodiversity is reduced; Group II consisting of the subgrouping with Combretum nigricans and
\end{abstract}


Microchloa indica and the subgrouping with Combretum micranthum and Gardenia sokotensis floristically rich, with however a regeneration dominated by Guiera senegalensis; grouping with Loudetia togoensis and Acacia macrostachya, in good state of conservation. Conclusion: The study made it possible to characterize floristically the various landscapes along the rainfall and anthropic gradient.

Thus, in view of the structures of the stands observed, the distribution of floristic diversity increases according to a gradient of decreasing aridity from the periphery to the internal part of the reserve. From the north to the south there is a degraded part, a part gradually invaded by Guiera senegalensis and another well preserved. This work, which provides an overview of the state of conservation of the floristic diversity of the Niger W Biosphere Reserve, allows taking appropriate measures to safeguard our plant resources.

Keywords: Floristic diversity, plateaus, Biosphere Reserve, Niger

\section{Résumé}

Introduction : $\mathrm{Au}$ Niger, la dégradation des écosystèmes induit une perte de la biodiversité et une accentuation des effets néfastes du changement climatique surtout au niveau des écosystèmes contractés des plateaux de la réserve de biosphère du $\mathrm{W}$ du Niger. Objectif : L'objectif de cette étude est de caractériser la variabilité spatiale de la flore suivant le gradient pluviométrique Nord- sud de la réserve. Méthodologie : Des relevés phytosociologiques, une classification hiérarchique ascendante et une analyse canonique des correspondances entre groupements végétaux et facteurs du milieu. Résultats-discussion : Les 126 espèces inventoriées comptent 88 dicotylédones, 28 espèces ligneuses, et 98 herbacées. Les familles les plus rencontrées sont: les Poaceae (21,42\%), les Fabaceae (12,69\%), les Rubiaceae $(7,14 \%)$ et les Convolvulaceae $(6,34 \%)$. Le spectre biologique est dominé par les thérophytes puis les microphanérophytes. La chorologie mondiale est marquée par les espèces africaines suivies des paléo-tropicales ou des pantropicales, et au niveau africain, les espèces soudano-zambéziennes sont les plus fréquentes. On note la présence d'espèces intermédiaires (GC-SZ et GC-SZ-Sah-S). Du nord au sud, 3 groupements végétaux ont été mis en évidence : le groupement à Sida cordifolia et Guiera senegalensis dont la phytodiversité est réduite; le groupement II formé du sous-groupement à Combretum nigricans et Microchloa indica et du sous-Groupement à Combretum micranthum et Gardenia sokotensis floristiquement riches, avec cependant une régénération dominée par Guiera senegalensis; le groupement à Loudetia togoensis et Acacia macrostachya, en bon état de conservation. Conclusion : L'étude a permis de caractériser sur le plan floristique les différents paysage le long du gradient pluviométrique et anthropique. Ainsi, 
au vu des structures des peuplements observés la distribution de la diversité floristique augmente suivant un gradient d'aridité décroissante de la périphérie vers la partie interne de la réserve. On distingue du nord au sud une partie dégradée, une partie progressivement envahie par Guiera senegalensis et une autre bien conservée.

Ce travail qui donne un aperçu sur l'état de conservation de la diversité floristique de la réserve de biosphère du $\mathrm{W}$ du Niger permet de prendre les mesures appropriées pour la sauvegarde de nos ressources végétales.

Mots-clés : Diversité floristique, plateaux, Réserve de Biosphère, Niger

\section{Introduction}

Les milieux semi-arides sont caractérisés par la précarité de leurs conditions environnementales qui fragilise l'équilibre des écosystèmes végétaux (Ouédrago et al, 2006). La distribution des ligneux est fortement liée à la latitude qui impose des conditions climatiques variables (Boussim et al, 1998). La longue saison sèche, les fluctuations climatiques et les fortes pressions anthropiques rendent la régénération ligneuse plus aléatoire en zone sahélo-soudanienne (Boussim et al, 1998 ; Gijsbers et al, 1994), notamment la reproduction par semis naturel (Bationo et al, 2005). Au Niger, la physionomie de la végétation varie du nord au sud à l'image de la pluviométrie qui augmente selon le même gradient (Saadou, 1990). La dégradation des écosystèmes induit une perte de la biodiversité et une accentuation des effets néfastes du changement climatique, situation qui compromet la durabilité des systèmes de production et expose les communautés locales aux calamités naturelles et à l'insécurité alimentaire (Nellemann et Corcoran, 2010). La situation est aujourd'hui encore plus préoccupante car l'exploitation forestière se pratique en ignorant les principes d'aménagement forestier durable. Le prélèvement sélectif de certaines espèces de bois d'œuvre de qualité menace dangereusement les ressources phytogénétiques du pays. Toutes ces perturbations entraînent une diminution de la biodiversité notamment la biodiversité végétale.

Ce processus de dégradation est important sur les plateaux, où les sols sont peu fertiles et n'offrent pas de conditions de développement favorables pour la végétation. Le cas de la réserve totale de faune de Tamou en est un exemple. En effet, depuis sa création en 1962, elle constitue une zone d'attraction pour les éleveurs en quête de pâturages et de l'eau pour la survie de leur bétail en saison sèche (Douma et al, 2012). Vaste espace de transition situé dans l'ouest du pays, réparti entre les zones sud-sahélienne et nordsoudanienne, la réserve de biosphère du $\mathrm{W}$ est essentiellement constituée de plateaux. Elle est d'une grande importance car renferme $80 \%$ de la biodiversité totale du Niger (Saadou, 1998) et joue un rôle socio-économique 
particulièrement considérable. Elle fournit aux populations de la nourriture, des fibres pour le textile, du bois, des médicaments à travers les plantes médicinales utilisées dans la médecine traditionnelle, etc.....Elle doit à ce titre bénéficier d'une protection.

Il est donc nécessaire de préserver l'intégrité des écosystèmes de la Réserve de Biosphère dont la fonction de sauvegarde est l'une des principales missions. L'efficacité de cette protection s'apprécie entre autres par l'étude de la diversité végétale.

La grande sécheresse des années 1972- 1973 a entrainé un déplacement massif des agriculteurs sédentaires du Nord vers cette périphérie du Parc National W du Niger. Cette occupation s'est traduit par le développement de défrichement illégal, de pastoralisme illégal et de braconnage et une extension des champs de culture (Ambouta, 2000) ce qui a beaucoup contribué à accentuer la dégradation des terres et la perturbation de ses écosystèmes.

Malgré l'importance dévolue de la richesse floristique de la réserve de biosphère du $\mathrm{W}$ du Niger, l'on ne dispose pas d'informations scientifiques et techniques sur sa diversité floristique depuis son occupation. Aucun travail d'envergure nationale n'a été réalisé dans ce sens. Les travaux réalisés par Douma et al, (2012) sont localisés autour des villages et ne décrivent que l'état de dégradation des quelques espèces emblématiques des parcs agro forestiers. Le processus de dégradation de la végétation a été constaté mais le lien avec les facteurs déterminants n'a pas été établi (Kebbede, 1988; Haile, 1990; Hulme, 1992).

Les travaux de Manzo (1996) ont mis en évidence des facteurs favorisant la dégradation de l'environnement notamment les actions anthropiques.

Pour mieux apprécier l'état de conservation de cette zone, il est important de connaître sa situation actuelle et suivre son évolution.

Le but de cette étude est de caractériser la variabilité spatiale de la flore du nord au sud de la réserve où la pluviométrie est abondante.

L'hypothèse de départ du présent travail repose sur le fait que la dégradation des conditions pluviométriques et les pressions liées aux activités humaines se répercutent sur la composition floristique et sur la distribution des espèces.

\section{Matériel et méthodes}

Site d'étude : l'étude a porté sur les formations contractées des plateaux de la fraction déclassée de la réserve totale de faune de Tamou, et de la zone tampon de la réserve de biosphère du $\mathrm{W}$ du Niger. Cette zone est située entre $12^{\circ} 28^{\prime}$ et $12^{\circ} 50^{\prime}$ latitude Nord et entre $2^{\circ} 06^{\prime}$ et $2^{\circ} 24^{\prime}$ longitude Est (Figure 1). Dans la zone, la pluviométrie est variable dans l'espace et dans le temps. Il s'agit d'un climat tropical semi-aride de type sahélien au Nord, où les 
précipitations sont de l'ordre de 400 à $600 \mathrm{~mm}$ et un climat tropical humide de type soudanien au sud où le régime pluviométrique avoisine les $800 \mathrm{~mm}$. Les plus fortes pluviométries sont enregistrées dans le mois d'août. A partir des données disponibles à la station de la Tapoa, il a été possible de mettre en évidence l'existence des années sèches et des années humides pendant la période 1981-2008.

Les indices pluviométriques des 27 dernières années évoluent en dent de scie et le nombre d'années sèches est plus élevé que celui des années humides, ce qui explique une tendance à la baisse des pluies (Douma, 2009). La température moyenne annuelle se situe autour de $36,7^{\circ} \mathrm{C}$. Le maximum de températures moyennes mensuelles se situe en avril avec $42^{\circ} \mathrm{C}$ et le minimum en décembre et février $\left(20^{\circ} \mathrm{C}\right)$. L'humidité relative est comprise entre $23 \%$ en avril (mois le plus sec) et 80,5\% en août (mois le plus humide) (Amadou, 2005).

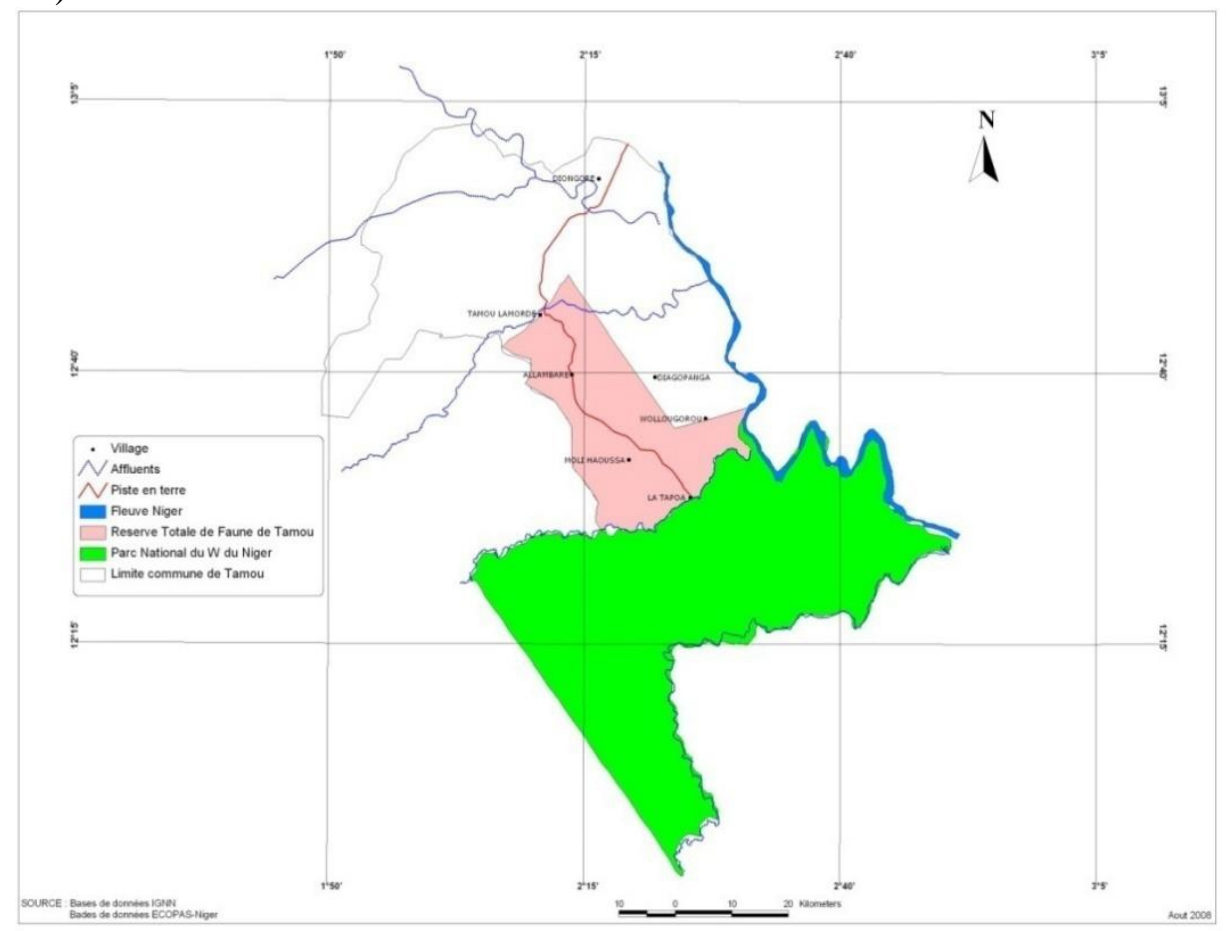

Figure 1 : Situation géographique de la zone d'étude

\section{Échantillonnage et relevés}

Les données pour caractériser la variabilité spatiale de la flore après des années d'occupations par les immigrés victimes de sécheresse, ont été obtenues par inventaires forestiers. Les unités d'observation sont des placettes de $1000 \mathrm{~m}^{2}(50 \mathrm{~m} \times 20 \mathrm{~m})$ délimitées le long d'un transect Nord-Sud. L'inventaire a été effectué sur 40 placettes, distantes de $500 \mathrm{~m}$ les unes des autres, soit un taux d'échantillonnage de $0,1 \%$.Les mesures effectuées portent 
sur les descripteurs des facteurs du milieu. Pour chaque plateau de $(50 \mathrm{~m}$ x20 m) observé, nous avons procédé :

(1) à l'identification de l'unité géomorphologique (plateau, versant, bas versant, bas fond, plaine, bourrelet sableux);

(2) à la description des états de surface du sol (Casenave et Valentin, 1990). Ils définissent la surface élémentaire comme une unité pédologique superficielle différenciée sur un même type de sol dont la surface est caractérisée par un couvert végétal homogène et une organisation qui résulte des transformations subies sous l'effet des facteurs météorologiques, phytoécologiques, fauniques et anthropiques. La typologie des surfaces élémentaires repose sur un certain nombre de critères caractéristiques du milieu et qui influencent la capacité d'infiltration. Les principaux critères sont le type de croûtes (9 types principaux), la nature des milieux, la charge grossière, l'activité faunique, les éléments modulateurs (prend en compte la couverture végétale, le microrelief, la texture du sol) et la porosité vésiculaire ;

(3) à la détermination de la texture du sol: La texture renseigne sur la composition du squelette et de son réseau poreux.

(4) la détermination de la profondeur du sol nécessite le recours à un profil pédologique. En ce qui concerne cette étude, l'information est obtenue en enfonçant une tige métallique à l'aide d'un marteau. La tige est ensuite défoncée et la profondeur est obtenue en mesurant la partie enfoncée. Cette technique a été recommandée par Mahamane et Saadou (2008);

(5) à l'estimation de la présence de blocs de cuirasse, de blocs de grès, de gravillons ;

(6) à l'appréciation de l'activité biologique (placage de termites, termitières). Elle a une influence directe sur l'infiltration par la porosité qu'elle génère. Cette porosité communique avec la surface, facilitant ainsi le drainage rapide de l'eau, de plus elle détruit au moins localement, les croûtes qui s'opposent à l'infiltration. (Casenave, 1991).

Des relevés phytosociologiques selon l'échelle d'abondancedominance de Braun Blanquet (1932) sont effectués en utilisant des documents de référence pour déterminer les espèces (Berhaut 1967 ; Saadou, 1998, Von Maydell, 1983). L'échelle est la suivante :

$+: 1 \%$ de recouvrement ou espèce présente à l'état d'individus isolés abondance et recouvrement très faibles

1 : Espèce présente à l'état d'individus peu nombreux qui occupent moins de $5 \%$ de la surface 
2 : Espèce présente à l'état d'individus abondants, recouvrement de 5 à $25 \%$ de la surface

3 : Espèce dont le recouvrement se situe entre 25 et $50 \%$

4 : Espèce dont le recouvrement se situe entre 50 et $75 \%$

5 : Espèce dont le recouvrement se situe entre 75 et $100 \%$.

\section{Analyse des données}

Les paramètres retenus pour caractériser la diversité floristique sont la diversité beta et alfa, les groupements végétaux, les spectres biologique et phytogéographique des espèces, la densité et la régénération des arbres.

Pour se faire les logiciels suivants ont été utilisés :

-le logiciel MVSP (Kovach, 2003) avec la classification hiérarchique ascendante (CHA), en comparant les relevés entre eux pour les regrouper en fonction de leur similarité. L'identification des groupements végétaux du milieu étudié a été faite avec la méthode de Braun Blanquet combinée à ce logiciel. La diversité béta est ensuite évaluée en calculant l'indice de diversité béta à travers le coefficient de Sorensen ou encore Dice Index(en abrégé D.I.) selon la formule:

$$
S=\frac{2 a}{2 a+b+c}
$$

$\mathrm{a}=$ nombre d'espèces communes à deux relevés $\mathrm{R} 1 \mathrm{et} \mathrm{R} 2 ; \mathrm{b}=$ nombre d'espèces appartenant au relevé $\mathrm{R} 1$ uniquement ; $\mathrm{c}=$ nombre d'espèces présentes dans le relevé $\mathrm{R} 2$ uniquement.

Le coefficient de Sorensen exprimé en pourcentage varie de 0 à $100 \%$ et permet de comparer les relevés entre eux pour les regrouper en fonction de leur similarité. Les résultats sont exprimés par un dendrogramme.

-le logiciel Excel pour le relevé phytosociologique, la taxonomie, le spectre biologique selon Raunkiaer, le spectre phytogéographique dans le monde et en Afrique, la densité des ligneux, l'indice de diversité alpha qui se calcule selon la formule :

$$
H=-\sum_{1}^{n} p_{i} \log _{2} p_{i}
$$

$\mathrm{pi}=$ proportion relative $\mathrm{du}$ recouvrement moyen de l'espèce $\mathrm{i}$ dans le groupement.

L'indice de Shannon varie de 0,5 à 4,5 et augmente avec la taille de l'échantillon.

\section{Résultats}

\section{Structure spécifique du peuplement}

La figure 3 montre la classification réalisée sur 40 relevés. 


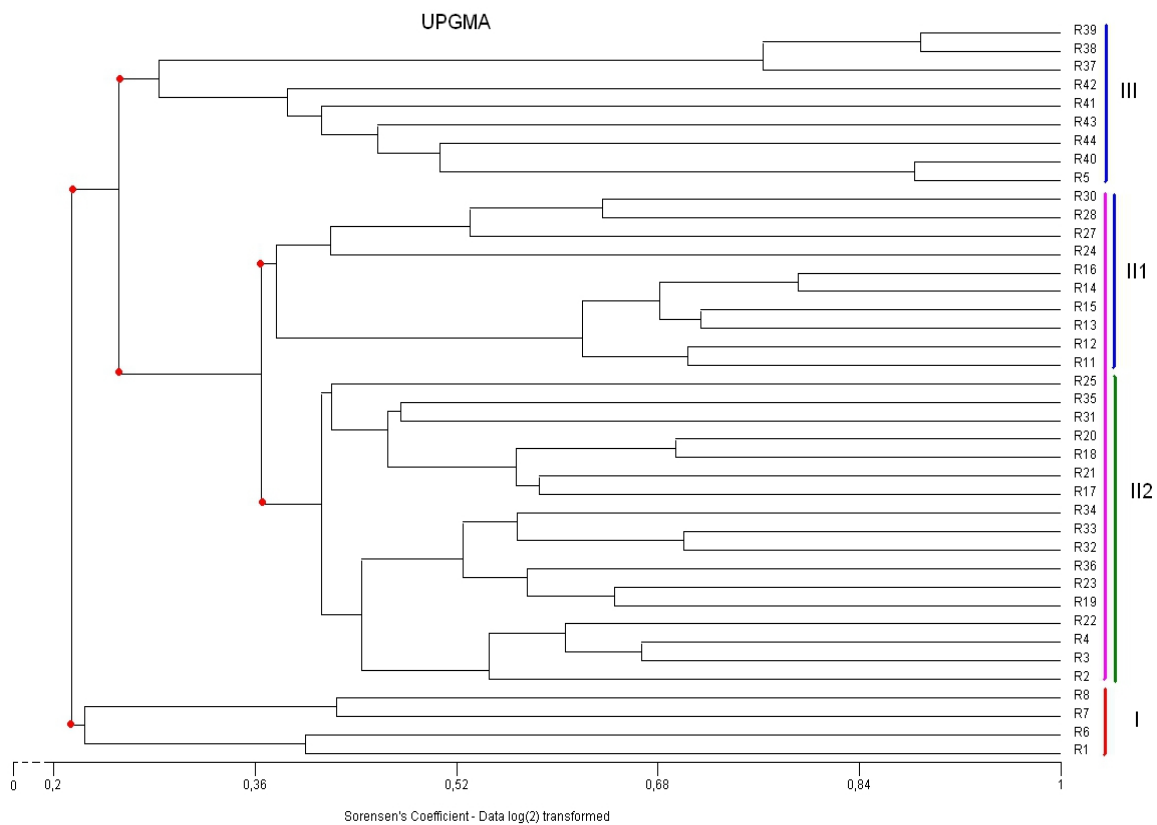

Figure 3 : Classification hiérarchique ascendante de 40 relevés

Au seuil de similarité de $22 \%$, trois groupes de relevés ont été obtenus : groupe I formé des relevés de l'extrême nord de la réserve ou les conditions pédoclimatiques sont peu favorables; groupe II formé d'un mélange des relevés de la partie nord plus aride moins arrosée (II1) et sud moins aride et plus arrosée (II2); groupe III concerne des relevés de la zone Tampon.

\section{Caractéristiques des groupements}

Sur les 40 relevés, 126 espèces ont été recensées dont 98 herbacées et 28 ligneux, appartenant à 89 genres et 32 familles.

Les dicotylédones représentent $84,38 \%$ des familles et $71,43 \%$ des espèces contre $15,62 \%$ des familles et $28,57 \%$ des espèces pour les monocotylédones.

Le groupement I a une diversité systématique de 33 espèces dont 6 ligneux et 24 herbacées. Ces espèces sont aussi dominées par les dicotylédones (88,24\% des familles) ; il est caractérisé par une abondance de Sida cordifolia et Guiera senegalensis.

Le sous-groupement II1compte 67 espèces, parmi lesquelles 13 ligneux et 54 herbacées. Les espèces dominantes sont Combretum nigricans et Microchloa indica. Dans ce sous-groupement les espèces sont réparties dans 27 familles et 55 genres. Les dicotylédones comptent 21 familles soit 77,77\% des familles alors que seulement 22,22\% des familles appartiennent à la classe des monocotylédones. 
La composition systématique du sous-groupement II2 est de 61 espèces dont 18 ligneux et 43 herbacées. Combretum micranthum et Gardenia sokotensis sont les espèces les plus caractéristiques de ce milieu. Les espèces de ce sous-groupement sont réparties en 48 genres et 25 familles; les dicotylédones représentent $84 \%$ des familles et les monocotylédones $16 \%$.

Le groupement III, marqué par la plus grande fréquence de Loudetia togoensis et Acacia macrostachya renferme 50 espèces parmi lesquelles 18 ligneux et 32 herbacées; $80 \%$ des espèces sont des dicotylédones, les monocotylédones représentent $20 \%$.

La liste des espèces rencontrées et celle des espèces caractéristiques pour l'ensemble des parcelles et pour chaque groupement ou sous-groupement est donnée par les tableaux I (a) et I(b).Tableau I (a) : Composition floristique des parcelles

\begin{tabular}{|c|c|c|}
\hline Gpt & Esp rencontrées & Espèces dominantes \\
\hline I & $\begin{array}{l}\text { Acanthospermum hispidum; Achyrantes } \\
\text { Alysicarpus ovalifolius; Aristida mutabilis; Aristida } \\
\text { stipoïdes; } \\
\text { Balanites aegyptiaca; Boscia senegalensis; Bracharia } \\
\text { xantholeuca; Cassia mimosoïdes; Combretum glutinosum; } \\
\text { Combretum micranthum; Corchorus tridens; Ctenium } \\
\text { elegans; Dactyloctenium aegyptium; Digitaria gayana; } \\
\text { Diheteropogon hageroupi; Fimbrustilis hispidula; Guiera } \\
\text { senegalensis; Hoslundia opposita; Loudetia togoensis; } \\
\text { Melliniea micrantha; Meremia pinnata; Microchloa indica; } \\
\text { Mitracarpus villosus; Mollugo nudicaulis; } \\
\text { Pergularia tomentosa; Piliostigma reticulatum; Polycarpea } \\
\text { linearis; Schoenopheldia gracilis; Sida cordifolia; } \\
\text { Spermacoce scabra; Waltheria indica; Zornia glochidiata }\end{array}$ & $\begin{array}{lr}\text { Sida } & \text { cordifolia; } \\
\text { Guiera senegalensis. }\end{array}$ \\
\hline III & $\begin{array}{l}\text { Acacia ataxacantha; } \text { Acanthospermum } \\
\text { Andropogon gayanus; Anthephora nigritana; Aristida } \\
\text { longiflora; Aristida mutabilis; Aristida stipoïdes; Asparagus } \\
\text { africanus; Balanites aegyptiaca; Boscia angustifolia; Boscia } \\
\text { senegalensis; Brachari axantholeuca; Caralluma } \\
\text { adscendens; Cassia mimosoïdes; Cassia obtusifolia; Cassia } \\
\text { sieberiana;Celosia trigyna; Combretum micranthum; } \\
\text { Combretum nigricans; Commelina benghalense; } \\
\text { Commelina forskaleii; Crotalaria atrorubens; Croton } \\
\text { gratissimus; Cyanotis lanata; Cyperus amabilis; Digitaria } \\
\text { gayana; Diheteropogon hageroupi; Dipcadi spp; } \\
\text { Endostemon tereticaulis; Eragrostis pilosa; Eragrostis } \\
\text { tenella; Eragrostis tremula; Evolvulus alcinioïdes; } \\
\text { Fimbrustilis hispidula; Gardenia sokotensis; Gloriosa } \\
\text { superba; Guiera senegalensis; Hoslundia opposita; }\end{array}$ & $\begin{array}{l}\text { Combretum nigrican; } \\
\text { Microchloa indica }\end{array}$ \\
\hline
\end{tabular}




\begin{tabular}{|c|c|c|}
\hline & $\begin{array}{l}\text { Indigofera bracteolata; Indigofera astragalina; Ipomea } \\
\text { dichroa; Ipomoea involucrata; Ipomoea vagans; Jacmontia } \\
\text { tamniflora; Leucas martinisensis; Loudetia togoensis; } \\
\text { Macrotyloma africanum; Melliniea micrantha; Meremia } \\
\text { pinnata; Microchloa indica;Mitracarpus villosus; Mollugo } \\
\text { nudicaulis; Mukia maderespatana; Pandiaca involucrata; } \\
\text { Pennisetum pedicellatum; Peristrophe paniculata; } \\
\text { Piliostigma reticulatum; Setaria sphaeolata; Sida cordifolia; } \\
\text { Spermacoce scabra; Sterculia setigera; Tridax procumbens; } \\
\text { Triumpheta pentandra; Zornia glochidiata. }\end{array}$ & \\
\hline III & $\begin{array}{l}\text { Acacia ataxacantha; Acacia macrostachya; } \\
\text { adscensionis; Aristida } \\
\text { Asparagus africanus; Balanites aegyptiaca; Bauhinia } \\
\text { rufescens; Boscia angustifolia; }\end{array}$ & $\begin{array}{l}\text { Combretum } \\
\text { micranthum; } \\
\text { Gardenia sokotensis }\end{array}$ \\
\hline
\end{tabular}

Tableau I(b): Composition floristique des parcelles

\begin{tabular}{|c|c|c|}
\hline $\mathrm{Gpt}$ & Esp rencontrées & Espèces dominantes \\
\hline $\begin{array}{c}\text { II2 } \\
\text { (suite) }\end{array}$ & $\begin{array}{l}\text { Boscia senegalensis; Cassia mimosoüdes; Cassia } \\
\text { obtusifolia; Citrilus lanatus; Combretum glutinosum; } \\
\text { Combretum micranthum; Combretum nigricans; } \\
\text { Crotalaria atrorubens; Crotalaria pallida; Cyanotis } \\
\text { lanata; Dactyloctenium aegyptium; Digitaria } \\
\text { horizontalis; Diheteropogon hageroupi; Endostemon } \\
\text { tereticaulis; Eragrostis tremula; Euphorbia hirta; } \\
\text { Evolvulus alcinioüdes; Fimbrustilis hispidula; Gardenia } \\
\text { aquala; Gardenia sokotensis; Grewia flavescens; Guiera } \\
\text { senegalensis; Hoslundia opposita; Indigofera leprieurii; } \\
\text { Ipomea kotschyana; Kyllinga squamulata; Lannea acida; } \\
\text { Lannea microcarpa; Loudetia togoensis;Meremi } \\
\text { apinnata;Microchlo aindica;Mitracarpus villosus; } \\
\text { Mollugo nudicaulis; Pandiaca involucrata; Pennisetum } \\
\text { pedicellatum; Peristrophe paniculata; Piliostigma } \\
\text { reticulatum; Polycarpea linearis; Polygala multiflora; } \\
\text { Schizachyrium exile; Schoenopheldia gracilis; Sida alba; } \\
\text { Sidac ordifolia; Spermacoce filifolia; Spermacoce } \\
\text { radiata; Spermacoce scabra; Tinnea barteri; Triumfetta } \\
\text { pentandra; Waltheria indica; Wissadula amplissima; } \\
\text { Ziziphus mucronata; Zornia glochidiata. }\end{array}$ & $\begin{array}{l}\text { Combretum } \\
\text { micranthum; } \\
\text { Gardenia sokotensis }\end{array}$ \\
\hline III & $\begin{array}{l}\text { Acacia ataxacantha; Acacia macrostachya; Ampelocissus } \\
\text { africana; Andropogon gayanus; Anogeissus leiocarpus; } \\
\text { Asparagus africanus; Aspilia kotschyi; Bidens biternata; } \\
\text { Bombax costatum; Boscia angustifolia; Boscia } \\
\text { senegalensis; Brachiaria ramosa; Cassia sieberiana; } \\
\text { Combretum glutinosum; Combretum micranthum; } \\
\text { Combretum nigricans; }\end{array}$ & $\begin{array}{l}\text { Loudetia togoensis; } \\
\text { Acacia } \\
\text { macrostachya }\end{array}$ \\
\hline
\end{tabular}




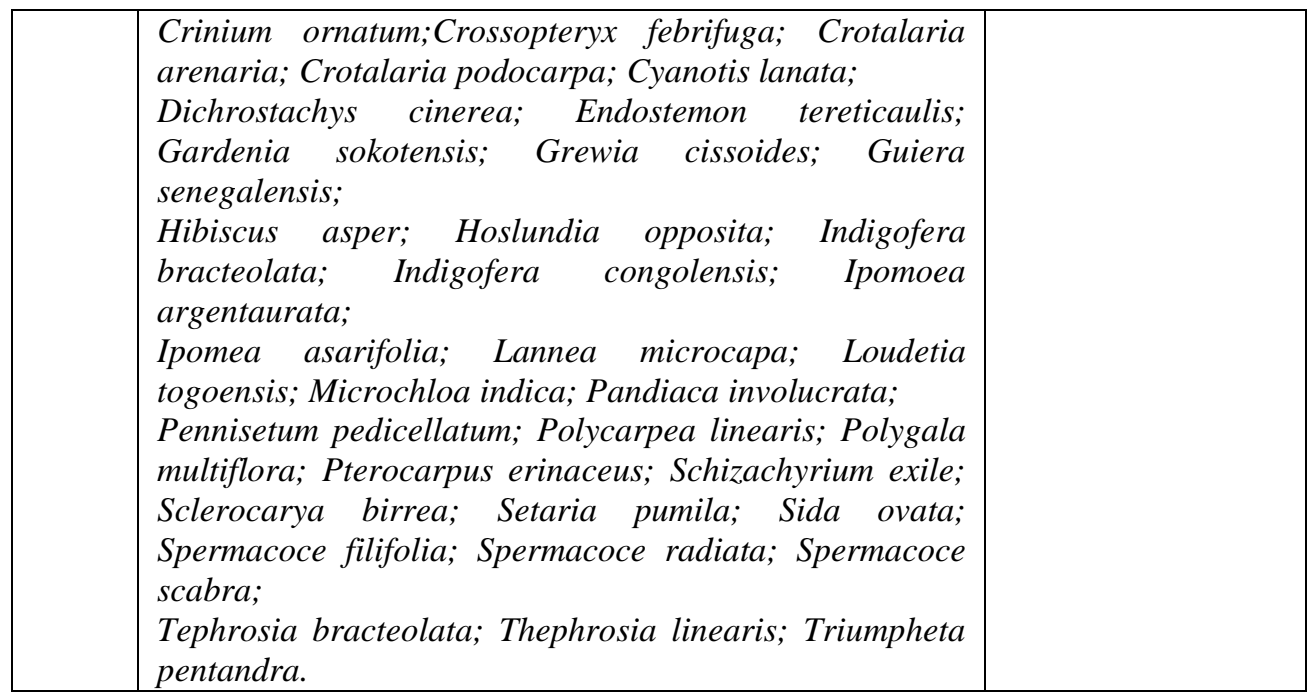

Le tableau II illustre la composition systématique des espèces pour les 40 relevés et pour chaque groupement ou sous- groupement.

Tableau II : Répartition taxonomique des espèces

\begin{tabular}{|c|c|c|c|c|c|c|}
\hline \multirow[t]{2}{*}{ Classe } & \multicolumn{2}{|c|}{ Famille } & \multicolumn{2}{|c|}{ Genre } & \multicolumn{2}{|c|}{ Espèce } \\
\hline & Nombre & $\%$ & Nombre & $\%$ & Nombre & $\%$ \\
\hline \multicolumn{7}{|c|}{ Ensemble } \\
\hline Dicotylédone & 28 & 87,5 & 69 & 76,66 & 90 & 71,43 \\
\hline Monocotylédone & 4 & 12,5 & 21 & 23,34 & 36 & 28,57 \\
\hline \multicolumn{7}{|c|}{ Groupement I } \\
\hline Dicotylédone & 15 & 88,24 & 20 & 62,5 & 21 & 63,64 \\
\hline Monocotylédone & 2 & 11,76 & 12 & 37,5 & 12 & 36,36 \\
\hline \multicolumn{7}{|c|}{ Sous-Groupement II1 } \\
\hline Dicotylédone & 21 & 77,78 & 36,00 & 65,45 & 43,00 & 64,18 \\
\hline Monocotylédone & 6 & 22,22 & 19,00 & 34,55 & 24,00 & 35,82 \\
\hline \multicolumn{7}{|c|}{ Sous-Groupement II2 } \\
\hline Dicotylédone & 21 & 84 & 34 & 0,71 & 45 & 73,77 \\
\hline Monocotylédone & 4 & 16 & 4 & 0,29 & 16 & 26,23 \\
\hline \multicolumn{7}{|c|}{ Groupement III } \\
\hline Dicotylédone & 18 & 81,82 & 30 & 75 & 40 & 80 \\
\hline Monocotylédone & 4 & 18,18 & 10 & 25 & 10 & 20 \\
\hline
\end{tabular}

\section{Diversité alpha}

L'indice de diversité alpha varie entre 2,619 pour le groupement I qui enregistre le plus faible indice, et 4,135 pour le sous-groupement II1. Le sousgroupement II1 a 3,731 et le groupement III 3,12.

Cependant, l'équitabilité des espèces au sein du groupement I est moyenne (coefficient d'équitabilité : 0,52 ) de même que celle du groupement 
III $(0,552)$ alors qu'elle est assez élevée pour les sous-groupements II1 $(0,681)$ et II2 $(0,629)$ (tableau III).

Tableau III : Diversité alpha des groupements

\begin{tabular}{|c|c|c|}
\hline Groupements & Indicede diversité & Coefficient d'équitabilité \\
\hline I & & 0,52 \\
\hline II1 & 2,619 & 0,681 \\
\hline II2 & 4,135 & 0,629 \\
\hline III & 3,731 & 0,552 \\
\hline
\end{tabular}

\section{Spectre biologique}

Ensemble des relevés : les thérophytes et les microphanérophytes sont les types biologiques les mieux représentés.

Groupement I : dans le spectre brut, les thérophytes occupent la première place $(69,7 \%$ des espèces). Les microphanérophytes viennent en seconde position avec $18 \%$ des espèces. Du point de vue recouvrement ce sont les thérophytes $(50,94 \%)$ et les chaméphytes $(42,49 \%)$ qui dominent.

Groupement II1 : Les spectres brût et pondéré montrent que les thérophytes (avec respectivement $56,72 \%$ et $48,78 \%$ des espèces) sont de loin les plus représentés. Les microphanerophytes viennent en deuxième position $(16,42 \%$ et $38,07 \%)$. Groupement II2: les thérophytes et les microphanerophytes occupent la première place avec respectivement 59,01\% et $24,59 \%$ des espèces selon le spectre brut, puis $51,81 \%$ et $45,92 \%$ sur le spectre pondéré.

Groupement III : à l'instar de autres groupements, les thérophytes et les microphanérophytes sont les types biologiques caractéristiques (tableau III).

Tableau III : Spectre biologique des espèces

\begin{tabular}{|c|c|c|c|c|c|}
\hline Groupement & \multirow{2}{*}{ TB } & \multicolumn{2}{|c|}{ Spectre brut } & \multicolumn{2}{c|}{ Spectre pondéré } \\
\cline { 3 - 6 } & & Nombre & Pourcentage & Nombre & Pourcentage \\
\hline \multirow{2}{*}{ Ensemble } & $\mathrm{mp}$ & 27 & 21,43 & 21,16 & 35,86 \\
\cline { 2 - 6 } & $\mathrm{T}$ & 70 & 55,6 & 32,39 & 54,89 \\
\hline \multirow{3}{*}{$\mathrm{I}$} & $\mathrm{T}$ & 23 & 69,7 & 27,13 & 50,94 \\
\cline { 2 - 6 } & $\mathrm{mp}$ & 6 & 18,18 & 2,88 & 5,4 \\
\cline { 2 - 6 } & $\mathrm{CH}$ & 1 & 3,03 & 22,63 & 42,49 \\
\hline \multirow{2}{*}{ III } & $\mathrm{T}$ & 38 & 56,72 & 32,1 & 48,78 \\
\cline { 2 - 6 } & $\mathrm{mp}$ & 11 & 16,42 & 25,05 & 38,07 \\
\hline \multirow{2}{*}{$\mathrm{II} 2$} & $\mathrm{~T}$ & 36 & 59,01 & 33,68 & 51,86 \\
\hline & $\mathrm{mp}$ & 15 & 24,59 & 29,82 & 45,49 \\
\hline \multirow{3}{*}{ III } & $\mathrm{T}$ & 26 & 52 & 44,22 & 68,03 \\
\cline { 2 - 6 } & $\mathrm{mp}$ & 16 & 32 & 17,78 & 27,35 \\
\hline
\end{tabular}




\section{Spectre phytogéographique}

\section{a. Affinités chorologiques dans le monde}

Les espèces africaines puis les pantropicales et les paléotropicales sont les plus importantes avec respectivement $61,11 \%, 16,67 \%, 15,87$.

Groupement I: dans le spectre brut, les espèces Africaines sont majoritaires (57,57\% de l'ensemble), suivies des Paléotropicales (21,21\%). Le spectre pondéré montre une prédominance des Pantropicales $(59,15 \%)$ puis des Africaines (36,62\%).

Groupement II1 : les espèces africaines sont au premier rang avec $59,7 \%$ dans le spectre brût et $69,75 \%$ dans le spectre pondéré (tableau 12). Les espèces paléotropicales représentent $16,42 \%$ des espèces au niveau du spectre brut mais dans le spectre pondéré elles viennent en troisième position $(9,95 \%)$ après les pantropicales $(18,31 \%)$. Groupement II2 : Les espèces africaines sont les plus représentées $(63,93 \%)$ sur le spectre brut et $75,95 \%$ sur le spectre pondéré. Les paléotropicales et les pantropicales ont une même représentativité dans le spectre brut $(16,39 \%)$, mais au niveau du spectre pondéré les pantropicales dominent avec $20,6 \%$ contre $3,35 \%$ pour les paléotropicales.

Groupement III : Les espèces africaines sont caractéristiques de ce groupe ( $84 \%$ dans le spectre brut et $88,72 \%$ dans le spectre pondéré).Les espèces à large répartition géographique sont faiblement représentées (tableau IV).

Tableau IV : Spectre phytogéographique dans le monde

\begin{tabular}{|c|c|c|c|c|}
\hline \multicolumn{5}{|c|}{ Ensemble } \\
\hline Spectre & \multicolumn{2}{c|}{ Prut } & Nombre & $\%$ \\
\hline TP & Nombre & $\%$ & 43,28 & 73,36 \\
\hline A & 77 & 61,11 & 12,22 & 20,7 \\
\hline $\mathrm{Pt}$ & 21 & 16,67 & 3,14 & 5,32 \\
\hline $\mathrm{Pal}$ & 20 & 15,87 & 14 \\
\hline \multicolumn{5}{|c|}{ Groupement I } \\
\hline $\mathrm{A}$ & 19 & 57,58 & 19,5 & 36,62 \\
\hline $\mathrm{Pt}$ & 5 & 15,15 & 31,5 & 59,15 \\
\hline $\mathrm{Pal}$ & 7 & 21,21 & 2 & 3,76 \\
\hline \multicolumn{5}{|c|}{ Sous-groupement III } \\
\hline $\mathrm{A}$ & 40 & 59,7 & 45,9 & 69,76 \\
\hline $\mathrm{Pt}$ & 11 & 16,42 & 6,55 & 9,95 \\
\hline $\mathrm{Pal}$ & 10 & 14,93 & 12,05 & 18,31 \\
\hline $\mathrm{A}$ & 39 & 63,93 & 49,32 & 75,95 \\
\hline $\mathrm{Pt}$ & 10 & 16,39 & 18,38 & 20,61 \\
\hline $\mathrm{Pal}$ & 10 & 16,39 & 2,18 & 3,35 \\
\hline \multicolumn{5}{|c|}{ Groupement III } \\
\hline $\mathrm{A}$ & 42 & 84 & 57,67 & 88,72 \\
\hline $\mathrm{Pt}$ & 3 & 6 & 4,78 & 7,35 \\
\hline
\end{tabular}




\section{b. Affinités chorologiques en Afrique}

Groupement I: Dans les deux spectres les guinéo-congolaisessoudano-zambeziennes occupent les premières places, suivies des soudanozambéziennes.

Groupement II1 : Les deux spectres montrent une nette dominance des soudano-zambéziennes $(40,29 \%$ pour le brut et $57,37 \%$ pour le spectre pondéré), puis des guinéo-congolaises-soudano-zambéziennes $(38,8 \%$ et $28,41 \%$ ).

Groupement II2 : Le spectre brut est caractérisé par un taux élevé des Guinéo-Congolaises-Soudano-Zambéziennes (37,7\%), puis des SoudanoZambéziennes $(31,14 \%)$. Sur le spectre pondéré les tendances sont inversées, $62,22 \%$ de Soudano-Zambéziennes contre 27,94\% de Guinéo-CongolaisesSoudano-Zambéziennes.

Groupement III : Les soudano-zambéziennes font $50 \%$ des espèces selon le spectre brut et $68,55 \%$ d'après le spectre pondéré. Les Guinéocongolaises- soudano-zambéziennes viennent en second ordre dans le spectre brut (28\% des espèces). Par contre dans le spectre pondéré ce sont les guinéocongolaises-soudano-zambéziennes-saharo-sindiennes qui occupent la deuxième place avec $18,97 \%$ des espèces (Tableau V).

Tableau V : Chorologie africaine

\begin{tabular}{|c|c|c|c|c|}
\hline \multicolumn{5}{|c|}{ Ensemble } \\
\hline \multirow[b]{2}{*}{ TP } & \multicolumn{2}{|c|}{ Spectre brut } & \multicolumn{2}{|c|}{ Spectre pondéré } \\
\hline & nbre & $\%$ & nbre & $\%$ \\
\hline SZ & 45 & 35,71 & 33,82 & 57,32 \\
\hline GC-SZ & 48 & 38,1 & 17,19 & 29,14 \\
\hline SZ-Sah.S & 16 & 12,7 & 1,8 & 3,04 \\
\hline GC-SZ-Sah.S & 15 & 11,9 & 6,08 & 10,30 \\
\hline \multicolumn{5}{|c|}{ Groupement I } \\
\hline GC-SZ & 12 & 36,36 & 46,13 & 86,62 \\
\hline $\mathrm{SZ}$ & 10 & 30,30 & 4,75 & 8,92 \\
\hline SZ-Sah.S & 6 & 18,18 & 0,75 & 1,41 \\
\hline GC-SZ-Sah.S & 4 & 12,12 & 1,50 & 2,82 \\
\hline \multicolumn{5}{|c|}{ Sous-groupement II1 } \\
\hline SZ & 27 & 40,3 & 37,75 & 57,38 \\
\hline GC-SZ-Sah.S & 7 & 10,45 & 8 & 12,16 \\
\hline GC-SZ & 26 & 38,80 & 18,7 & 28,42 \\
\hline \multicolumn{5}{|c|}{ Sous-groupement II2 } \\
\hline SZ & 19 & 31,15 & 40,41 & 62,23 \\
\hline
\end{tabular}




\begin{tabular}{|c|c|c|c|c|}
\hline & & & & \\
\hline GC-SZ & 23 & 37,70 & 18,15 & 27,94 \\
\hline SZ-Sah.S & 10 & 16,39 & 2,50 & 3,85 \\
\hline GC-SZ-Sah.S & 9 & 14,75 & 3,88 & 5,98 \\
\hline \multicolumn{5}{|c|}{ Groupement III } \\
\hline SZ & 25 & 50 & 44,56 & 68,55 \\
\hline GC-SZ & 14 & 28 & 5,89 & 9,06 \\
\hline GC-SZ-Sah.S & 5 & 10 & 12,33 & 18,97 \\
\hline
\end{tabular}

\section{Densité et taux de régénération des ligneux}

La densité des ligneux est de 182 pieds/ha ; la régénération ligneuse du groupement I est formée de jeunes pousses de Guiera senegalensis, avec un taux de $19,23 \%$.

Dans le groupement II1, la densité des ligneux est de 1228 pieds/ha ; Guiera senegalensis et Gardenia sokotensis constituent l'essentiel de la régénération ; le taux de régénération est de 16,61\%.

La densité des ligneux du groupement II2 est de 493 pieds/ha, la régénération est plus importante pour Guiera senegalensis, Grewia flavescens, Combretum micranthum, C. nigricans et Gardenia sokotensis; le taux de régénération est de $73 \%$.

Dans le groupe III la densité des ligneux est de 974 pieds/ha ; les jeunes pieds sont dominés par Acacia macrostachya, Guiera senegalensis, Combretum micranthum, Combretum nigricans et Gardenia sokotensis ; le taux de régénération est de $52,15 \%$.

\section{Discussion et conclusion}

\section{Caractéristiques de la flore}

Les résultats de cette étude fournissent des informations importantes sur la variation de la diversité floristique des plateaux de la Réserve de Biosphère du W du Niger. Sur l'ensemble des relevés, nous avons recensé. 126 espèces.

Ces dernières sont dominées par les Poaceae (21,42\%), Fabaceae (12,69\%), Rubiaceae $(7,14 \%)$ et Convolvulaceae $(6,34 \%)$. Ces résultats concordent avec ceux de Mahamane (2005) dans le parc du W qui signale que les familles les plus importantes de la flore sont les Poaceae, suivies des Fabaceae, des Cyperaceae et des Asteraceae. Cette dominance des graminées peut être considérée comme un indice de pression, puisque le bétail exerce une 
consommation sélective de la phytomasse. Aussi, les graminées sont des espèces qui résistent aux différentes perturbations. Ceci a été déjà évoqué Saidou et al (2010) sur la végétation de la station de Toukounous. Tiendrebeogo et Sorg (1997) au Burkina Faso expliquent cet état de fait par le piétinement des animaux en saison des pluies provoquant d'abord un tassement de l'horizon superficiel peu profond. Selon Burel et Baudry (1999) jusqu'à un certain seuil, les perturbations augmentent la diversité d'un écosystème. Dans le même ordre d'idée, Barot et al (1999), Henkel (2003) indiquent que les forte perturbations en zones périphériques tendent à une diversification, sont très faibles ou quasi nulles en zone intérieure qui tend vers une homogénéisation.

Dans cette végétation les thérophytes et les microphanérophytes constituent les types biologiques les mieux représentés, ce qui signifie que la majorité des plantes des espèces traversent la longue saison sèche sous forme de graine, les parties végétatives étant détruites par dessiccation. Ces résultats sont similaires à ceux obtenus par Amani (2008) dans la région de Maradi. Ceci pourrait être dû à la longue période sèche que les thérophytes semblent bien supporter en prolongeant et en bouclant leur cycle de développement pendant la saison humide. Tout se passe comme si le système racinaire de ces types biologiques notamment les microphanérophytes arrive au niveau de la nappe phréatique durant la période sèche, ce qui leur permet de satisfaire leurs besoins en eau.

Les affinités chorologiques mondiales montrent que les espèces africaines sont les plus rencontrées, suivies des paléotropicales et des pantropicales. Elles correspondent effectivement à la zone étudiée; mais les recouvrements moyens montrent souvent que les pantropicales, espèces à large distribution géographique dépassent les paléotropicales; ceci pourrait être dû à l'influence des activités humaines sur le milieu.

Concernant la chorologie africaine, les espèces soudano-zambeziennes sont les plus représentées car caractéristiques de la zone (Saadou, 1990). Elles sont suivies des guinéo-congolaises-soudano-zambéziennes qui selon le même auteur constituent un élément de liaison entre les climats équatorial et soudanien. Les espèces soudano-zambéziennes-saharo-sindiennes et les GCSZ-Sah.S sont rencontrées dans tous les groupes en faibles proportion, témoins de la proximité de la zone saharo-sindienne. Notre zone d'étude est située à la limite nord de la zone soudano-zambezienne. Certaines espèces typiquement Sahélienne se retrouvent de plus en plus en zone soudanienne (Douma, 2016), témoins d'une modification des conditions locales particulières d'ordre microclimatique, édaphique et anthropique. Les espèces ligneuses les plus fréquentes sont: Guiera senegalensis, Combretum micranthum, Gardenia sokotensis. Elles sont représentées généralement par de jeunes individus issus de rejet de souches, des drageons ou des marcottes et, au même moment $C$. 
nigricans présente des vieux sujets. Dans ces conditions, une régression de l'espèce $C$. nigricans est possible dans le temps, au profit de G. senegalensis à l'image du phénomène observe au niveau de la végétation du plateau situé plus au nord. En effet selon Mahamane (2005) l'association à C. nigricans et G. senegalensis évolue généralement vers un appauvrissement en Combretum nigricans et une formation de forêts basses constituées essentiellement de $G$. senegalensis.

En plus des phénomènes climatiques et de la pression sur les terres, un autre facteur peut être mis en cause pour expliquer cette régression : il s'agit de l'exploitation accrue de $C$. nigricans comme bois de chauffe par les populations, or des études similaires ont montré que l'exploitation frauduleuse et excessive du bois, couplée à la pression agricole et pastorale et à celle de l'usage du bois comme énergie domestique compromet la pérennité de ces faciès forestiers (Dourma, 2012).

Combretum glutinosum, Balanites aegyptiaca, Boscia senegalensis, Piliostigma reticulatum, Anogeissus leiocarpa, Lannea microcarpa, Pterocarpus erinaceus semblent subir les conséquences de cette anthropisation, elles sont très peu présentées. D’après Barmo (2008), ces plantes sont beaucoup utilisées comme bois-énergie, dans la construction des habitats, l'artisanat ou dans la médecine traditionnelle. Selon le même auteur certaines de ces plantes sont devenues rares ou en voie de disparition : Anogeissus leiocarpus, Lannea microcarpa, Pterocarpus erinaceus. Parmi les 126 espèces végétales recensées, 54 soit 42,8\% ont été citées comme plantes médicinales (Mamadou, 2017).

D’après Mahamane (2005), le phénomène de sécheresse et de désertification a engendré d'importantes vagues de mortalité au niveau de la strate arborée dans le parc du W; de très nombreux pieds morts de Prosopis africana, Anogeissus leiocarpa, Pterocarpus erinaceus ou Terminalia macroptera ont été retrouvés.

\section{Caractéristiques des groupements}

Le groupement à Sida cordifolia et Guiera senegalensis se trouve sur un sol peu profond (profondeur inférieure à $0,5 \mathrm{~m}$ ) à texture gravillonnaire ou sableuse avec une mosaïque des buttes de sables, témoin de l'érosion et des plages nues assez importantes. Cette structure est régie par la topographie, le substrat édaphique et l'humidité relative du sol. Il se distingue par l'abondance de Sida cordifolia et Guiera senegalensis ce qui traduit probablement l'état de dégradation du sol, ces espèces poussant généralement sur sol pauvre. La présence de buttes expliquerait l'abondance de Zornia glochidiata, espèce pionnière caractéristique des sols dunaires. Nous avons cependant noté la présence de quelques croûtes de décantation et croûtes biologiques et aussi des placages de termites et de rares termitières. L'indice de diversité alpha $(2,62)$ 
est faible, le coefficient d'équitabilité $(0,52)$. Le recouvrement moyen des pantropicales est majoritaire $(59,15 \%$ du recouvrement moyen total), ce qui mettrait en relief la forte pression anthropique subie par ce milieu.

Le sous-groupement à Combretum nigricans et Microchloa indica sur sol à texture sableuse de profondeur variable (parfois supérieure à $1 \mathrm{~m}$ ) avec une croûte structurale à 3 horizons, de pente faible, de texture sableuse est floristiquement le plus riche de notre étude. L'état de surface présente beaucoup de litière, une croûte biologique importante avec placage de termites et des termitières.

La diversité alpha $(4,13)$ est élevée, de même que la répartition des espèces (coefficient d'équitabilité de 0,68). D’après Savadogo et al. (2007), un indice de diversité élevé correspond à une grande égalité des contributions individuelles au couvert végétal. L'augmentation de la pression anthropique durant la saison sèche est responsable des changements de la composition de la végétation (Metzger et al, 2005). La densité des ligneux est de 1228 pieds/ha mais le taux de régénération est faible (16,61\%). Guiera senegalensis est l'espèce la plus représentée parmi les jeunes pieds. Selon Douma et al (2012) dans cette zone, le défrichement se fait sans dessouchage et la reconstitution de la végétation ligneuse s'effectue à partir des souches et des racines : source de rejets et de drageons. Dans le même ordre d'idée, ces auteurs indiquent que c'est la grande capacité reproductive des Combretaceae (forte aptitude à la multiplication par voie végétative) qui pourrait être à l'origine de leur forte représentativité par rapport à d'autres plantes dans la zone déclassée.

Le sous-groupement à Combretum micranthum et Gardenia sokotensis évolue sur un sol peu profond à texture sablo-limono-argileuse, marqué par des croûtes de décantation, des croûtes d'érosion, présentant des gravillons latéritiques, quelques fois des blocs de cuirasse voire un affleurement de la roche-mère et souvent des plages nues. La topographie montre une pente moyenne à faible ; la diversité alpha de 3,731 est moins élevée que celle du sous-groupe II1, la densité des ligneux est de 493 pieds/ha, la régénération surtout importante pour Guiera senegalensis; le taux de régénération est de $73 \%$; mais l'équitabilité des espèces dans le groupement est bonne $(0,63)$. Il pourrait donc s'agir d'un groupement initialement à Combretum micranthum, C. nigricans et Gardenia sokotensis sur un sol subissant actuellement une érosion accompagnée d'un envahissement progressif par Guiera senegalensis et d'une diminution de la phytodiversité du milieu. La présence de l'espèce Gardenia aquala, malgré sa faible proportion, indique l'état d'appauvrissement du sol. Gardenia sokotensis est généralement retrouvé sous le couvert des Combretum. Cette espèce pousse habituellement sur sol humide trouve des conditions favorables grâce au microclimat des couverts notamment l'accroissement de la fertilité du sol sous ombrage elle-même associée à 
différents facteurs qui sont entre autres l'apport de litière par l'arbre et l'herbe (Bernhard-Reversat,1982, 1986), l'apport d'éléments par les précipitations (Kellman, 1979), la redistribution d'éléments nutritifs en surface à partir des horizons profonds (Bosch et Van Wyk 1970) et l'apport d'éléments fertilisants par les animaux domestiques (Akpo, 1973) et les oiseaux (Tréca et al.,1996).

Le groupement à Loudetia togoensis et Acacia macrostachya, évolue sur un sol de profondeur variable de texture limono-argileuse avec des blocs de cuirasse du bois mort, de la litière et une croûte biologique ainsi que des placages de termites. La similitude entre un relevé de du Nord (R5) et ceux de la zone tampon pourrait s'expliquer par le fait qu'en dépit des modifications de la composition et la structure de la végétation au Nord la végétation authentique subsiste encore dans certaines stations. Le bois mort retrouvé serait probablement en relation avec la forte mortalité de certains ligneux observée ces dernières années dans le parc du W (Mahamane, 2005). L'espèce ligneuse caractéristique de ce milieu est $C$. nigricans; les herbacées y sont abondantes en l'occuence $L$. togoensis, S. exile et $M$. indica ; la diversité alpha $(3,12)$ est plus élevée que celle du groupement à Sida cordifolia et Guiera senegalensis, le coefficient d'équitabilité est moyen $(0,552)$; la densité des ligneux est assez élevée (974 pieds/ha); les jeunes pieds sont dominés principalement par Acacia macrostachya; le taux de régénération est élevé $(52,15 \%)$.

$\mathrm{Au}$ fur et à mesure que l'on avance des zones périphériques moins arrosée mais plus anthropisé vers la partie centrale de la réserve plus arrosée et moins anthropisé, il y a une certaine discorde entre la végétation et le régime pluviométrique. Elle est probablement liée à l'état physique du sol ; il y a souvent un affleurement de la roche-mère rendant le sol peu ou pas épais à certains endroits. Cependant, l'état de conservation de ce milieu et le renouvellement de la strate ligneuse semblent bons : il n'y a pas de signes d'impact des activités humaines, l'absence totale d'espèces à large distribution géographique en est un indicateur.

L'étude a contribué à une meilleure connaissance de la diversité floristique de la végétation contractée des plateaux de la partie de la réserve déclassée. Elle a permis de ressortir que les Poaceae $(21,42 \%)$ et les Fabaceae $(12,69 \%)$ sont dominantes. Trois groupes de relevés se distinguent plus ou moins nettement :

- le premier est un groupement à Sida cordifolia et Guiera senegalensis évoluant sur un plateau dénudé au Nord, ayant la plus faible pluviométrie où le sol est de texture gravillonnaire ou sableuse et la phytodiversité globalement réduite;

- les deux autres sont les sous-groupements II1 et II2 situés à la périphérie de la réserve (Tamou) et la zone tampon (groupement III) ou zone 
de transition (Moli). C'est à ce niveau que se trouve le groupement à Loudetia togoensis et Acacia macrostachya le mieux conservé de l'étude.

L'évolution pourrait aboutir à un envahissement des plateaux par Guiera senegalensis, au détriment des autres espèces comme Combretum nigricans.

Cette étude a permis de caractériser la variation de la diversité floristique des plateaux de la réserve de biosphère du $\mathrm{W}$ en fonction du gradient Nord-Sud. Ainsi la diversité floristique mis à part les quelques poches de dégradation suit un gradient d'anthropisation décroissante au fur et à mesure que l'on quitte la périphérie pour la partie centrale de la réserve. Il y a une nette différence entre les zones anthropisées et celles qui sont moins anthropisées. Au vu des résultats obtenus, il se dégage des perspectives de recherche qui devraient être entreprises dans un proche avenir. Il s'agit notamment :

- du suivi périodique de cette végétation afin de suivre l'évolution du milieu, ce qui permettrait de mieux apprécier l'ampleur du phénomène de diminution de la diversité végétale, y compris des plantes médicinales ;

de quantifier le prélèvement de bois effectué à travers des enquêtes socioéconomiques sur ces espèces depuis la périphérie jusqu'à la zone tampon du parc ;

d'étudier les mécanismes de régénération naturelle et de mortalité, des principales espèces d'arbres pour mieux comprendre la dynamique de renouvellement naturel sur ces plateaux.

\section{References:}

1. Akpo L.E. (1993). Influence du couvert ligneux sur la structure et le fonctionnement de la strate herbacée en milieu sahélien. Paris: Orstom éditions, TDM $93 \mathrm{~F}$.

2. Amadou B., 2005. Observatoire de Tamou, Situation de la commune rurale de Tamou. Rapport technique ROSELT 65p.

3. Amani A. (2008). Biodiversité végétale des milieux de culture du souchet dans la région de Maradi (NIGER). Mémoire de DEA, Université Abdou Moumouni de Niamey, 82 p.

4. Ambouta J. M. K. (2000). Rapport analytique sur la connaissance de l'habitat des girafes du Niger. PURNKO/SNV, Niamey, 33p.

5. Barmo S. (2008). Analyse socio-économique de l'exploitation des ressources végétales de la Réserve Totale de Faune de Tamou (Niger), Mémoire de DEA, UAM de Niamey, 64 pages.

6. Barot S., Gignoux J., Menaut J. C., 1999. Seed shadows, survival and recruitment: how simple mechanisms lead to dynamics of population recruitment curves. Oikos, 86 (2): 320-330. 
7. Bationo B.A., Karim S., Bellefontaine R., et al. (2005). Le marcottage terrestre: une technique économique pour la régénération de certains ligneux tropicaux. Sécheresse en ligne, 1E (3). www.secheresse.info/article.php3?id_article $=2342$.

8. Berhaut J. (1967). Flore du Sénégal. 2ème édition plus complète avec les forêts humides de la Casamance, 485p.

9. Bernhard-Reversat F. (1982). Biogeochemical cycle of nitrogen in a semi-arid savanna. Oikos, $38: 321-32$.

10. Bernhard-Reversat F. (1986). Le recyclage des éléments minéraux par la strate herbacée dans un peuplement naturel à Acacia et dans une plantation d'Eucalyptus au Sénégal. Acta Ecol. Génér., 7: 353-64.

11. Bosch O.J., Van Wyk JP. (1970). The influence of bushwemd trees on the productivity of Panicum maximum a preliminary report. Hand Weidingsveren South Afr, 5: 69-70.

12. Boussim J., Gampine D., Ilboudo J.B. (1998). Étude des contraintes à la régénération naturelle de neuf espèces ligneuses du Burkina Faso. In : Bâ AT, Madsen J, Sambou B, eds. Végétation et biodiversité au Sahel. Danida: Aarhus.

13. Braun-Blanquet J. (1932). Plant sociology. The study of plant communities. Ed. McGray Hill, New York, London, 439 p.

14. Burel F., Baudry J. (1999). Écologie du paysage : Concepts, méthodes et applications. Paris, France, Technique et Documentation, 359 p.

15. Casenave A. \& Valentin C. (1990). Les états de surface de la zone sahélienne : influence sur l'infiltration. ORSTOM, (Collections Didactiques), Paris $280 \mathrm{p}$.

16. Casenave A. (1991). Spécificité de processus du ruissellement en zone sahélienne. Influence des états de surface. In Utilisation rationnelle de l'eau des petits bassins versants en zone aride. Ed AUPELF UREF.JohnLibbeyEurotext Paris, p77-86.

17. Douma S. (2009). Ecologie de quatre espèces ligneuses de la Réserve Totale de Faune de Tamou (Niger) : Indicateurs de gestion durable des ressources forestières. Mémoire de DEA, Université Abdou Moumouni de Niamey, Niger, 88p.

18. Douma S., Idrissa S., Morou B., Mamoudou H., Saley K., Mahamane A., Saadou M. (2012). Diversité du peuplement ligneux des parcs agroforestiers de la Réserve Totale de Faune de Tamou (Niger). Etudes et recherches sahéliennes, Sciences agronomiques, 18: 55 - 62 .

19. Douma S. (2016). Etude ethnobotanique et écologique des espèces ligneuses alimentaires de soudure des systèmes agroforestiers du sudouest du Niger: diversité, importance, structure et niveau de mesure. Thèse de Doctorat, Faculté des Sciences et Techniques/UAM, Niamey, 93p. 
20. Dourma M., Wala K., Kudzo A. G.,Ronald B., Philippe D., Sêmihinva A., Komlan B., Koffi A. (2012). Typologie, caractéristiques structurales et dynamique des faciès forestiers fragiles à Isoberlinia spp. en vue de leur gestion au Togo. Bois et forêts des tropiques, $\mathrm{N}^{\circ} 3$ 13 (3) :19-33

21. Gijsbers H.J.M., Kessler J.J., Knevel M.K. (1994). Dynamic and natural regeneration of woody species in farmed parklands in the Sahelian region (Province of Passore, Burkina Faso). For Ecol. Manage, 64: 1-12.

22. Haile. (1990). Drought in Ethiopia in WMO symposium on meteorological aspects of tropical droughts. 30 April- 4 May 1990. WMO TMRP, Niamey, series 1190, 36: 273-8.

23. Henkel T. W. (2003). Monodominance in the ectomycorrhizal Dicymbe corymbosa (Caesalpiniaceae) from Guyana. Journal of Tropical Ecology, 19: 417-437.

24. Hulme. (1992). Rainfall changes in Africa: 1931-1960 to 1961-1990. Int .J. climatol., 12: 685-99.

25. Kebbede. (1988).Cycles of famine in a country of plenty: the case of Ethiopia. Geojournal 17:125-3.

26. Kellman M. (1979). Soil enrichment by neo-tropical savanna trees. $J$ Ecol, 67: 565-77.

27. Kovach Computing Services. (2003). Multi-Variate Statistical Package. Version 3.1 Users' Manual, 137p.

28. Mahamane A. (2005). Etudes floristique, phytosociologique et phytogéographique de la Végétation du Parc Régional du W du Niger. Thèse de Doctorat en Sciences Agronomiques et Ingénierie Biologique, Université Libre de Bruxelles-Belgique, 520p.

29. Mahamane A. et Saadou M. (2008). Synthèse sur l'harmonisation des méthodes inventaire de la flore et de la végétation. $8 \mathrm{p}$.

30. Mahamane L. (2005). Dynamique de la végétation dans le domaine sahélien du Niger occidental suivant un gradient d'aridité : rôle des facteurs écologiques, sociaux et économiques ; Faculté de Sciences, Université Abdou Moumouni, Niamey, 186p.

31. Mamadou A. J., Saley K., Boubé M., Rokia S., Saadou M. (2017). Enquête ethnobotanique Auprès Des Tradipraticiens De Santé Des Régions de Niamey et Tillabéri Au Niger: Données 2012-2017. EuropeanScientific Journal, 13 (33) : 276.

32. Manzo M. (1996). Etude des jachères dans l'ouest nigérien, gestion traditionnelle et structure du peuplement végétal dans le canton de Torodi. Thèse de $3^{e ̀ m e}$ cycle, Université de Ouagadougou, 136p. 
33. Metzger K.L., Coughenour M.B., Reich R.M., Boone R.B. (2005). Effects of seasonal grazing on plant species diversity and vegetation structure in a semi-arid ecosystem. J Arid Environ, 61: 147-60.

34. Nellemann C. \& Corcoran E. (2010). Dead Planet, Living Planet Biodiversity and Ecosystem Restoration for Sustainable Development. A Rapid Response Assessment. United Nations Environment Programm. http://www.grida.no/publications/rr/deadplanet/ebook.aspx

35. Ouédrago A., Thiombiano A., Hahn- Hadjali K. et Guinko S. (2006). Diagnostic de l'état de dégradation des peuplements de quatre espèces ligneuses en zone soudanienne du Burkina Faso. Sécheresse, 17 (4): $485-491$.

36. Saadou M. (1990). La végétation des milieux drainés nigériens à l'Est du fleuve Niger. Thèse d'Etat, Université de Niamey, 395p.

37. Saadou M. (1998). Evaluation de la biodiversité biologique au Niger : éléments constitutifs de la biodiversité végétale. Conseil National de l'Environnement pour un Développement Durable SE/CNEDD. Projet NER/ 97 / G 31 / A / 1 G / 99 "Stratégie Nationale et plan d'action Diversité Biologique", 138p.

38. Saidou O., Douma S., Zakou Djibo A., Riccardo F. (2010). Analyse du peuplement herbacé de la station sahélienne expérimentale de Toukounous (Niger) : composition floristique et valeur pastorale. Sécheresse, 21 (2) : 154-60.

39. Savadogo P., Tigabou M., Sawadogo L. et al. (2007). Woody species composition, structure and diversity of vegetation patches of a Sudanian savanna in Burkina Faso. Bois et forêts des tropiques, 4: 5 20.

40. Tiendrebeogo JP, Sorg JP(1997). Etude de la capacité de charge de la forêt classée de Gonze. Ouagadougou (Burkina Faso) : GTZ.

41. Tréca B., Tamba S., Akpo L.E., Grouzis M. (1996). Importance de l'avifaune sur les apports en azote et en phosphore dans une savane sahélienne du Nord-Sénégal. Rev. d'Ecol., 51 : 359-73, (Terre et Vie).

42. Von Maydell H. J. (1983). Arbres et arbustes du sahel, leurs caractéristiques et leurs utilisations. G.T.Z., Schriftenreihe, Eschborn, Allemagne, 254p. 